\title{
Ombre de la guerre et guerre des ombres : remarques à propos de Jules César
}

Raphaëlle Costa de Beauregard

\section{(2) OpenEdition}

Journals

\section{Édition électronique}

URL : http://journals.openedition.org/shakespeare/1211

DOI : 10.4000/shakespeare.1211

ISSN : 2271-6424

Éditeur

Société Française Shakespeare

\section{Édition imprimée}

Date de publication : 1 novembre 1990

Pagination : 67-78

Référence électronique

Raphaëlle Costa de Beauregard, «Ombre de la guerre et guerre des ombres : remarques à propos de Jules César », Actes des congrès de la Société française Shakespeare [En ligne], 8| 1990, mis en ligne le 01 janvier 2007, consulté le 05 mai 2019. URL : http://journals.openedition.org/shakespeare/1211 ; DOI : 10.4000/shakespeare.1211 


\section{SHAKESPEARE \\ ET LA GUERRE}

Ouvrage publié avec le concours de la Commission

des Communautés Européennes 
Illustration de la couverture : DERRICKE'S Image of Ireland, 1586 


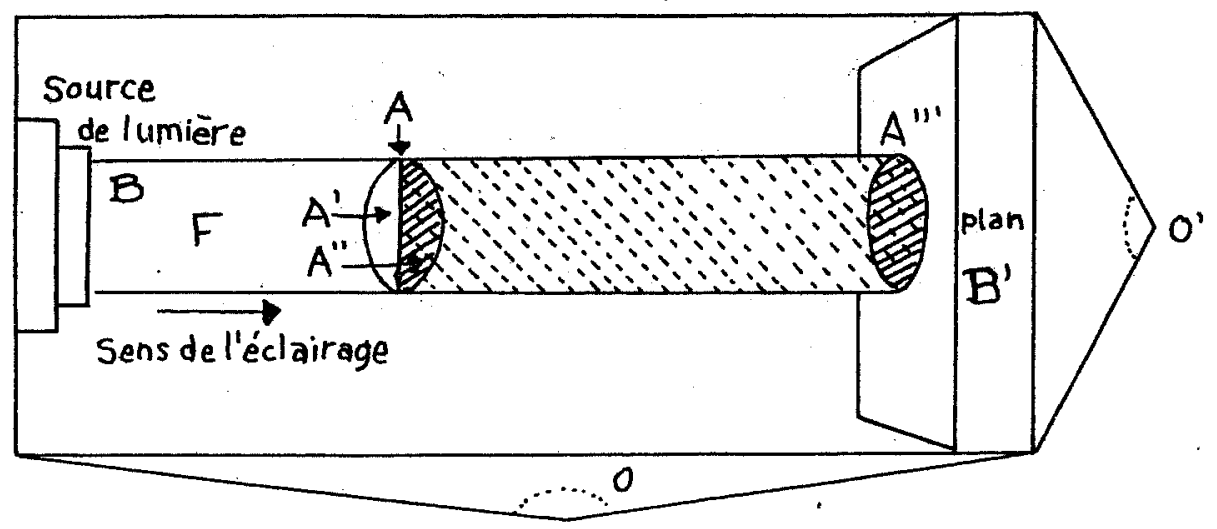

Fig. 1

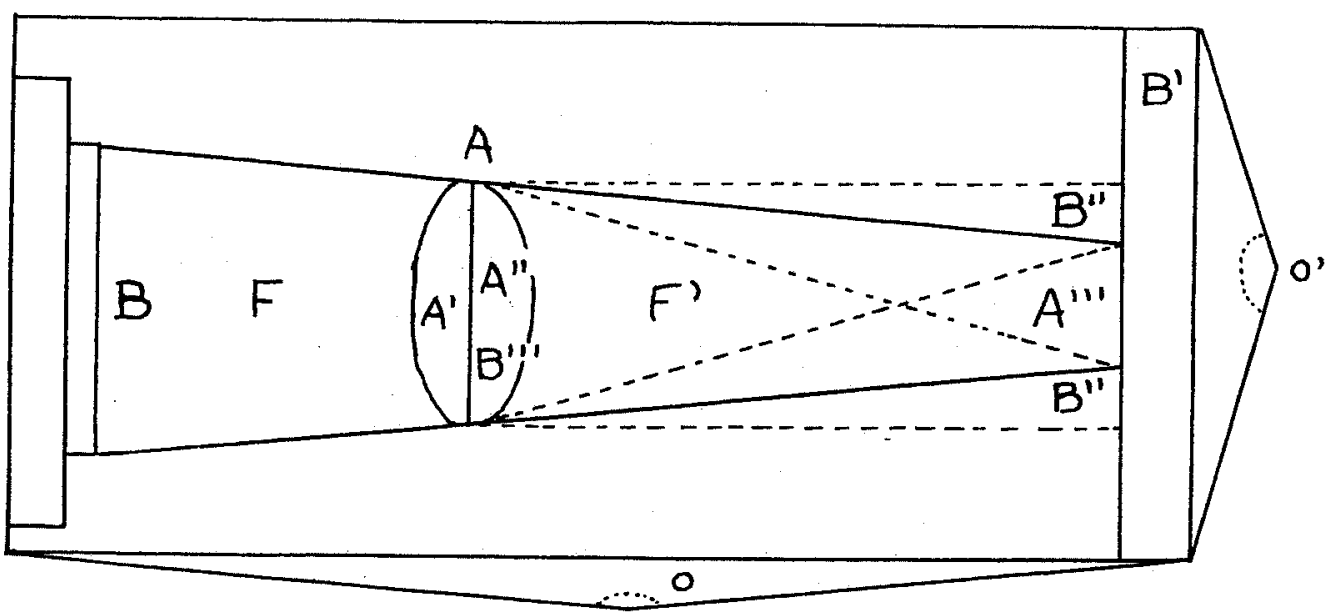

Fig.2

Dessins de l'auteur (d'après L. de Vinci)

Ombres de la guerre et guerre des ombres. 


\section{Ombres de la guerre et guerre des ombres: remarques à propos de Jules César ${ }^{1}$}

Le théâtre élisabéthain n'ayant pas de chœurª̀ qui la tâche d'une distanciation ou d'une mise en perspective ait pu être confiée, celle-ci est dévolue au fou. Ce point de vue différent, grâce auquel le spectateur peut voir chez les personnages ce qu'eux-mêmes ne peuvent soupçonner, est tellement essentiel au mécanisme dramatique que l'on rencontre d'autres manières de le produire telles que l'introduction d'un fantôme ou de sorcières. Dans Hamlet le fantôme donne au vécu l'aspect répugnant d'une trahison; dans Macbeth, les sorcières projettent sur les événements un sens nouveau, puisque ceux-ci deviennent la réalisation progressive d'une destinée déjà écrite. Ces procédés permettent l'instauration d'un monde parallèle, invisible, qui cadre le microcosme de la pièce et lui donne un sens universel.

En peinture, ce rôle est confié à l'éclairage. Dans les enluminures, c'est le sens des rayons d'or émanant de la partie supérieure du cadre, et désignant ainsi à l'observateur l'élu dans la foule; c'est aussi bien entendu celui de l'auréole, trace de ce rayon divin, qui valorise, et par conséquent nous fait évaluer, l'image ${ }^{3}$. Une fois l'éclairage maitrisé avec l'avènement du réalisme ${ }^{4}$, celui-ci s'adresse toujours à l'observateur, car la source lumineuse est latérale, dans le plan de la toile, et donc perpendiculaire aux rayons visuels émanant des yeux de celui-ci ${ }^{5}$. Même si la source hors-cadre reste longtemps située au-dessus du personnage, rappelant ainsi son origine céleste et divine, il s'agit dans le code même du réalisme d'imposer une réflexion et donc une distanciation critique par rapport au sujet. 
Ayant déjà cherché à montrer comment, dans Othello, une distance est établie entre le personnage d'Othello, cet étranger dont le visage obscur évoque à s'y méprendre un émouvant contre-jour, et celui de Iago, dont l'ombre indéchiffrable poursuit sa victime jusqu'aux tréfonds de son âme, je m'attacherai ici à étudier l'exploitation des stratégies du clair-obscur dans Jules César. La représentation de la guerre est le sujet central de cette tragédie, sous trois formes, dirait-on: la guerre larvée, potentielle, tout d'abord, au premier acte; puis la guerre illicite parce que parricide, déchaînant la guerre civile, et enfin une guerre qui se veut licite et vengeresse, entraînant le suicide de Brutus. Non seulement le texte montre que les ombres sont utilisées pour rehausser la tragédie, mais on pourrait aussi suggérer qu'elles permettent l'expression de la division intérieure, laquelle est présentée comme le germe de toute guerre.

Il y a là une innovation caractéristique du tournant du siècle. En effet, si l'on croit Hilliard ${ }^{6}$, miniaturiste d'Elisabeth I, l'ombre doit être rejetée comme une technique trompeuse et impure. Alors même que la mode du noir commençait à céder le pas à celle du blanc à la cour, on voit en même temps le clair-obscur apparaître chez Oliver $^{7}$, au grand dépit de Hilliard qui maintient son point de vue encore vers 1600:

if a very well favoured woman stand in a place where is great shadow, yet showeth she lovely; not because of the shadow, but because of her sweet favour... if she be not very fair... then shadow to show her in doeth her favor...»

Tandis que Donne et Essex préféraient faire faire leur portrait par Oliver ${ }^{9}$, Hilliard s'exprimait encore avec une formule lapidaire: «to shadow as if it were not at all shadowed is best shadowed ${ }^{10}$. Les valeurs de l'ombre telles que les définit Hilliard, et avec lui le Moyen Age finissant, sont: le mensonge, mais aussi le contour lorsqu'il s'agit 
d'ombre portée et le relief, obtenu par l'éclairage latéral: «shadows in pictures were indeed caused by the (...) coming in of the light only one way into the lace at some small high window ${ }^{11}$.

Or si Hilliard affiche un rejet du clair-obscur, il n'en reconnaît pas moins l'existence:

if one must be drawn or painted blowing the coal in the dark to light a candle, we must then shadow accordingly, making no light but that which comes from the coal... if one should make the troop of Judas going to seek Christ in the garden by night with torches and lanterns... the matter consisteth chiefly in the traitorous act done by night ${ }^{12}$

Je rappellerai brièvement le système des trois ombres tel que l'explique Léonard de Vinci, système dont le renouveau réaliste des frères Carrache et du Caravage devait tirer le parti que l'on sait, et cela dès 1595 . Georges de la Tour vers 1630-40 en fut le continuateur éminent ${ }^{3}$.

La première ombre, la plus noire, est celle que perçoit un observateur pour qui l'objet qu'il regarde est éclairé latéralement. (Fig. 1, A étant l'objet, l'ombre primitive A" est l'envers de A'). Pour un observateur non plus situé en 0 mais en 0 ', l'ombre primitive est ce que l'on appelle le contre-jour.

À cette ombre (A") dite primitive ou primaire, s'ajoute une ombre dérivée ou secondaire, dont le faisceau est symétrique et inverse par rapport au faisceau lumineux intercepté par le volume $A$. ( $\left.F \neq F^{\prime}\right)$.

Une troisième ombre, appelée A" pour souligner sa filiation avec l'objet A, est l'ombre portée sur un plan B', dont le contour est fidèle à A si le faisceau est perpendiculaire, et déformé s'il n'est pas perpendiculaire à B'.

Léonard de Vinci souligne ensuite l'importance des reflets, et des lumières qui viennent éclairer la face en contre-jour de manière à en rendre le relief visible. (Fig. 2 C'est le cas de la source lumineuse plus grande que l'objet 
A). Cette multiplication des reflets de lumière trouve sa contrepartie dans une multiplicité de reflets produits par les ombres:

par expérience, nous le constatons pour le soleil, tous les objets qui lui sont exposés participent de sa Iumière et le réfléchissent aux autres objets, comme on le voit pour la lune et autres étoiles qui nous renvoient la lumière que le soleil leur a donnée. Les ténèbres en font autant, revêtant de leur obscurité ce qui se renferme en elles ${ }^{14}$.

Ainsi pour l'observateur situé en 0', dans l'axe de l'ombre, l'acte de regarder devient une exploration de l'ombre pour elle-même, et l'acte de représenter consiste à faire voir dans l'ombre. Un grand nombre de portraits du dix-septième siècle paraissent ainsi extraire le personnage d'une pénombre qui sert de fond, un éclairage latéral ayant été ajouté et correspondant, pour l'observateur situé en 0 , à la position de 0 .

Une tradition différente émane également de ce contre-jour, c'est celle qui développe le cas imaginé par Léonard de Vinci où la source de lumière, $(B$, fig. 2) est plus petite que l'objet A. Dans ce cas l'objet projette une ombre $F$ '. beaucoup plus grande que ne l'est le faisceau $F$ issu de B; du point de vue de l'observateur situé en O', le contre-jour A" constitue un écran au premier plan derrière lequel il devine une source lumineuse plus petite. Ce type de contre-jour écran a donné chez Georges de la Tour de très beaux tableaux tels que L'Ange apparaissant à St Joseph ${ }^{15}$.

Quant au système des trois ombres combinées, et vues par un observateur situé à la fois en 0' (du fait d'un personnage en contre-jour au premier plan) et en $\mathrm{O}$, du fait de l'utilisation d'un éclairage latéral, il a donné lieu aux chefs d'œuvres que l'on sait; je rappellerai au passage La Cène à Emmaüss ${ }^{16}$ de Caravage, ainsi que Le Tricheur et La Diseuse de Bonne Aventure ${ }^{17}$ de Georges de la Tour, tous deux directement inspirés de toiles du Caravage trai- 
tant ces sujets. La différence essentielle entre Léonard et Titien, d'une part, et Caravage d'autre part, relève non seulement d'une inspiration lyrique chez les deux premiers et réaliste chez le dernier, mais aussi du choix du sujet: chez les premiers, ce sont la beauté et le message esthétique qui dominent, chez le dernier, ce sont la supercherie, le vol, le mensonge, c'est-à-dire en somme le conflit et la guerre entre les personnages qui sont traités. Dans toutes ces situations conflictuelles, le clair-obscur dramatise la vérité en la rendant évidente par une mise en perspective produite grâce au contre-jour et je propose de voir comment ces stratégies visuelles sont exploitées dans Jules César de manière à nous faire saisir des aspects de la connaissance tels que l'ambiguïté des apparences et l'aveuglement causé par la vanité. Il est en outre remarquable de voir le lien étroit qui existe au plan de ces stratégies visuelles entre les ombres et le miroir.

La troisième scène du premier acte de Jules César est nocturne, et c'est aussi le cas des deux premières scènes du deuxième acte. Cependant, avant d'être envahie par la nuit, la scène du premier acte est divisée en deux espaces diurnes, chacun défini par le lieu où se trouvent César d'une part et Brutus d'autre part. Les didascalies signalent cette rupture spatiale par des allées et venues ; or la simultanéité des deux actions serait mieux rendue si l'on imaginait deux zones, l'une, en pleine lumière et à l'arrièreplan, occupée par Jules César au Capitole refusant la couronne, et l'autre, dans l'ombre et au premier-plan, consacrée à la conversation entre Brutus et Cassius. Le texte nous dit en effet que tout Rome est accouru pour voir César, et Cassius compare un peu plus tard la foule de ses admirateurs à un bûcher allumé à la gloire du héros (Jules César, I.iii.109). Cette lumière est celle de la reconnaissance du héros par le public, ce que confirme la couronne. Lorsque sa destinée réelle est annoncée à César : «Beware the Ides of March», «What man is that?» «A soothsayer bids you beware the Ides of March » (I.ii.18-9), on ne peut s'empêcher de penser à la Diseuse de Bonne Aventure du 
Caravage $^{18}$. Dans ce tableau, la victime est en pleine lumière, tandis que la diseuse est en contre-jour. Or César se contente de dire «Set him before me, let me see his face». Tout porte à croire que César est ici un observateur ébloui par la lumière et aveuglé de ce fait au point de prendre l'apparence pour la réalité. (Cf. fig. 1, A' de A). Dans la mesure où l'objet donné à voir est la destinée, l'aveuglement de César est évident pour l'auditoire parce que celui-ci, en position $\mathrm{O}$, peut embrasser d'un même coup d'œil deux interprétations différentes de la destinée, celle, éclairée, où les apparences sont trompeuses, et celle, cachée, invisible pour César, où l'ombre recèle la vérité. L'opposition entre l'être et le paraître est dramatisée par l'inversion inattendue de son rapport avec la lumière et l'ombre.

Or simultanément la vérité nous est montrée en contre-jour, puisque Cassius s'adresse à Brutus pour l'associer au complot, tandis que celui-ci tourne délibérément le dos au Capitole et à la lumière dont il vient d'être question. On peut donc imaginer que l'auditoire puisse voir la vérité dans l'ombre. Par exemple, les rumeurs du Capitole atteignent Brutus de loin et sont l'occasion d'une erreur d'interprétation de sa part puisqu'il imagine le tyran que César sera à partir d'une réalité qu'il n'a pas vue . À la remarque de César: «let me see his face», fait écho celle de Cassius: «Can you see your face?» (I.ii 20 et 50) L'ombre où ils se trouvent est une zone où il est difficile de voir clair, où les amis peuvent se méprendre («let not therefore my good friends... construe any further my neglect...» 43-5). Brutus est lui aussi aveuglé, mais sur un mode très différent de l'aveuglement de César; ici, ce sont le doute, les questions, l'examen du miroir métaphorique que lui offre Cassius, qui figurent son aveuglement.

En outre le miroir offert par Cassius permet à Brutus de voir son ombre et non son visage: «mirror, as will turn/Your hidden worthiness into your eye,/That you might see your shadow» (56-8). Cette ombre «portée» (Fig. 1, A"') que Brutus doit voir dans le miroir, c'est l'ombre de l'ombre (Fig. 1, A"' est aussi l'ombre de A"); 
il l'associe immédiatement au danger: «Into what dangers would you lead me, Cassius?» (63), tandis qu'il vient de la définir: "poor Brutus, with himself at war,/Forgets the shows of love to other men» (46-7). C'est donc l'ombre de la guerre intérieure qui le mine, celle qu'il décide de cacher désormais: "O Conspiracy... Hide it [le visage] in smiles, and affability» (II.i.77-82).

Par ailleurs, de même que la diseuse de bonne aventure raconte son boniment en tenant en quelque sorte le regard de sa victime dans le miroir de son propre regard tout en lui faisant les poches, de même Cassius montre-t-il à Brutus son image dans le miroir métaphorique dont il parle afin de fixer son attention, tout en lui mentant sans vergogne puisqu'il le flatte: «I your glass,/will modestly discover to yourself/That of yourself which you yet know not of» (I.ii.68-70). Ces paroles ont pour but de retenir l'attention de la victime par un regard piégé. Cassius peut ensuite jouer sur les mots qu'il dépouille littéralement de leur sens dans l'usage pour leur donner un sens différent, entièrement lié au contexte de la conspiration: «I know that virtue to be in you, Brutus/As well as I do know your outward favour. / Well, Honour is the subject of my story » (90-2). L'on sait quel parti sera ensuite tiré du mot «honour» par Brutus puis par Antoine. La traîtrise de Cassius ici est ensuite confirmée lorsqu'il lui fait envoyer de faux messages secrets de la part de représentants imaginaires de l'opinion publique. Cassius en conclut en secret: «Well, Brutus, thou art noble: yet I see/Thy honourable mettle may be wrought/From that it is dippos'd» (305-7). La ruse mensongère est donc représentée de pair avec l'innocente naïveté, et l'auditoire une fois encore peut embrasser et réunir en un seul coup d'œil deux points de vue contradictoires. Deux modes de connaissance, l'un vu en contre-jour, comme en négatif: l'innocence (et l'ignorance de César un peu avant) et l'autre en positif: le mensonge (et le doute de Brutus avant) sont donc rendus visibles, ou tout au moins intelligibles, par des stratégies de caractère visuel et de structure analogue à celle du clairobscur en peinture. Ces différentes formes d'ambiguïté 
dépendent, pour leur représentation, en grande partie d'effets de distanciation dus à l'éclairage métaphorique que projette un énoncé sur le précédent.

Outre la représentation de la guerre intérieure et des relations entre les individus (César et le «prophète»; Brutus et Cassius), c'est aussi la guerre larvée que se livrent les factions dans Rome qui est ainsi dramatisée. En effet, dans la scène sur laquelle s'ouvre la tragédie, le triomphe de César est présenté comme un scandale, et c'est la figure du grand Pompée qui est évoquée. Or Pompée occupe une place centrale dans les scènes nocturnes que nous venons d'évoquer, car c'est à l'ombre de l'arc de Pompée que les conspirateurs se donnent rendez-vous, et c'est de cette même ombre qu'ils sont issus masqués par l'ombre de leur chapeaux. L'ombre de ce «porche» évoque d'une part l'ombre portée de Pompée assassiné quatre ans auparavant, et le souvenir que l'arc immortalise, et d'autre part l'ombre primitive, la face cachée d'où sort la vérité de la conspiration. Enfin c'est aussi l'ombre médiévale mensongère, car elle engendre des traîtres qui vont se servir du mensonge pour approcher César. Pour Brutus, la conspiration est un monstre issu de la nuit, «by night/When evils are most free» (II.i.78-9); l'ombre du «porche» de Pompée est à la fois signe de l'ambiguité des apparences et signe du mal.

Ainsi les valeurs médiévales associées par Hilliard à la nuit mensongère sont-elles juxtaposées avec la valeur réaliste du clair-obscur révélateur de la vérité.

En effet, la nuit ou plutôt la pénombre dont Léonard analyse le mécanisme n'est pas envisagée comme cachant la vérité mais au contraire comme la rendant visible:

Le clair-obscur ajoute une grande grâce au visage de ceux qui sont assis aux portes d'habitations sombres. Les yeux qui les regardent voient la partie ombragée de ces visages obscurcis par les ombres de l'habitation, et la partie éclairée de la clarté que lui donne la splendeur de l'air ${ }^{19}$. 
C'est bien de cette pénombre initiatrice qu'il s'agit dans les deux scènes nocturnes où Brutus veille en compagnie de Lucius (J.C., II.I et IV.ii). Tandis que la première fois Brutus reste aveugle devant la réalité, malgré la lumière de la chandelle qu'il fait allumer par Lucius pour remédier à la lumière insuffisante des étoiles, puisqu'il lit les messages qui lui sont envoyés sans deviner la supercherie, la deuxième fois, il est au contraire si lucide qu'il est le seul à voir le fantôme de César lorsque celui-ci se manifeste. Tandis que la pénombre reste impénétrable pour Brutus au début, elle est source de révélation à la fin de la pièce. On aura remarqué qu'ici il ne s'agit pas tellement d'ombre portée ou de contre-jour ponctuel, mais davantage de l'ensemble des ombres que projette un corps opaque éclairé par une source de lumière plus petite que lui. La chandelle de Lucius (et son nom bien entendu) évoque la petitesse de la source lumineuse, et rappelle des effets analogues obtenus en peinture, soit par Rembrandt plus tard, ou par Georges de la Tour avec Le Souffleur à la Lampe $^{20}$. Le rapport entre la source lumineuse et l'ombre envahissante est dramatisé dans ce cas: il y a comme un conflit entre les deux mondes, la chandelle symbolisant la connaissance rationnelle, celle qui organise le monde, la hiérarchise et la borne, en quelque sorte, tandis que l'ombre symbolise la connaissance occulte, irrationnelle, ou encore indirecte, faite de reflets, d'impressions fugitives et saisissantes. Ce type de conflit entre deux modes de connaissance est aussi dramatisé dans la peinture religieuse, mais dans ce cas, la lumière est du côté du divin et non du rationnel. (Cf. les nombreux Saint Jérôme au début du dix-septième siècle).

Outre la fonction initiatrice de l'ombre de César à Philippes, il semble que celle-ci soit aussi la réplique de celle de Pompée au début de la tragédie. Il semblerait difficile dès lors de voir dans la guerre punitive menée par Antoine et Octave telle qu'elle est représentée par Shakespeare une guerre véritablement licite. Bien au contraire, la division est signalée au moment même où ils sont le plus unis; en outre leur légitimité a été mise en 
doute par les allusions aux massacres ordonnés après le meurtre de César. En outre, si Octave parle de l'armée ennemie en termes militaires, afin de garantir la valeur du combat, Shakespeare donne la parole à Caton, fils de Caton d'Utique afin d'évoquer le camp de Pompée jusqu'au bout. En outre Cassius et Brutus se font justice eux-mêmes; aucune exécution punitive n'a lieu. Bien plus, la pièce se termine par l'éloge de Brutus que prononce Octave. Il semble que la paix ne soit qu'un armistice précaire entre deux ombres qui sont renvoyées dos à dos sans qu'une solution durable soit envisagée par Shakespeare.

Nous sommes donc renvoyés en définitive à la guerre intérieure que se livrent des devoirs incompatibles, guerre que Brutus seul a su assumer jusqu'au bout, comme le dit Antoine.

This was the noblest Roman of them all:

All the conspirators save only he,

Did that they did, in envy of great Ceaser:

He only, in a general honest thought,

And common good to all, made one of them. (V.v.68-72)

On peut se demander si ces devoirs contradictoires qui inspirent à Brutus le doute et l'erreur, mais aussi une certaine clairvoyance à la fin, ne sont pas des Idées au sens de Platon. Ne serait-ce pas là la véritable représentation de la guerre dans la pièce? Ce n'est plus de l'ombre de la guerre qu'il s'agit, ombre projetée par Pompée d'abord et par César ensuite, mais d'une guerre d'ombres, s'il est vrai que ce que nous croyons être des Idées ne sont que les ombres imparfaites d'une paix qui nous échappe toujours. 


\section{NOTES}

(1) Shakespeare Jules César, 1599. Les références sont celles de l'édition A. Harbage, Londres: Allen Lane the Penguin Press, 1969.

Rappelons à toutes fins utiles que cette tragédie inaugure un changement important puisque c'est la première des grandes tragédies, parmi lesquelles Hamlet et Macbeth lui font pour ainsi dire immédiatement suite. La communication sur Othello à laquelle il est fait allusion un peu plus loin est la suivante: «L'Othello de Shakespeare et la grammaire des ombres de Léonard de Vinci: réflexions sur une sémiotique de l'apparence», Nouvelles recherches en grammaire, Colloque d'Albi, Langages et Signification, 1987, Toulouse: P.U.M., 1988, pp. 241-81.

(2) Si l'on excepte le rôle du commentateur dans Henry $V$, lequel invite l'auditoire davantage à se représenter le lieu et le temps de l'action qu'à établir une distance critique.

(3) Pour les manuscrits, voir par exemple Philippe Laver, Les enluminures romanes, Paris, 1927 ou Eric G. Millar, La miniature anglaise aux XIV et $X V^{t}$ siècles, tr. Jean Buhot, Paris et Bruxelles: G. van Oest, 1928. Voir aussi les frères Limbourg, Les Très Riches Heures du duc de Berry, Chantilly: Musée Condé.

(4) IL s'agit ici essentiellement des trois techniques du réalisme en peinture: anatomie, perspective et proportions.

(5) Les rayons visuels étaient supposés analogues aux rayons lumineux: émis par une source, ils étaient reflétés par l'objet et renvoyés à la source. Cf. Martin Kemp, «Leonardo and the visual pyramid», Journal of the Warburg and Courtauld Institute, XC (1977), pp. 128-49.

(6) Nicholas Hilliard (1547-1619), The Arte of Limning, 1600 env. 1re éd. Philip Norman, Walpole Society, I (1912), 1-54. $2^{\mathrm{e}}$ éd. R.K.R. Thornton and T.G.S. Cain, A Treatise Concerning The Arte of Limning, Manchester: Mid Northumberland Arts Group and Carcanet New Press, 1981,139p. Le traîté a été traduit en français, cf. R. Costa de Beauregard Le portrait élisabéthain dans l'œuvre de Nicholas Hilliard, Thèse, Paris IV, 4/4/87, 3 vols., vol. 2

(7) Isaac Oliver, c. 1560-1617. Grand rival de Hilliard dans l'art du portrait en miniature à partir de 1590 environ, cet artiste d'origine rouennaise chercha à imposer à l'art de la miniature les techniques de la peinture réaliste qu'il possédait for bien : la perspective, l'anatomie et les proportions. En outre, il s'initia au clair-obscur. Cf. Roy Strong, The English Renaissance Miniature, Londres: Thames \& Hudson, 1983, pp. 142-85.

(8) Hilliard, p. 87. J'ai traduit ainsi: «Faire des hachures comme s'il n'y avait pas d'ombre est la meilleure façon de peindre». En effet, «to shadow» signifie peindre pour Hilliard; en outre, pour le miniaturiste, peindre c'est se servir d'un pinceau plus mince qu'une plume, et dessiner à l'aide de hachures.

(9) Roy Strong, p. 161.

(10) Hilliard, p. 87.

(11) Hilliard, pp. 86-7.

(12) Hilliard, p. 89.

(13) Léonard de Vinci, 1425-1519, Tratatto della Pittura di Lionard de Vinci... Rafaelle du Fresne..., Bologne, 1786. Ed. consultée, Paris: Jean de Bonnot, 1982, trad. André Keller. Les manuscrits de Léonard furent composés entre 1489 et 1518 . On sait que dès 1490 Léonard envisageait la 
publication du Traité de la peinture. Une première ébauche est citée par 'Luca Pacioli en 1498. Il en existait plusieurs copies en Italie et en Espagne, et ce texte était connu bien avant sa première publication en 1651. Le Titien, 1488-1576, Femme à sa toilette, Paris: Musée du Louvre, Le Caravage, 1573-1610; Georges de la Tour, 1593-1652.

(14) Léonard, 244, $\mathrm{n}^{\circ} 631$.

(15) Georges de la Tour, L'Ange apparaissant à St. Joseph, Nantes : Musée des Beaux-Arts. Reproduction en couleurs: Jacques Thuillier, Tout l'œuvre peint de Georges de La Tour, Paris: Flammarion, 1973, $2^{\mathrm{e}}$ éd. 1985, PL.XXXIX

(16) Le Caravage, La Cène à Emmaüs, Londres: National Gallery. Reproduction en couleur: Giorgio Bonsanti Le Caravage, Florence: Scala, 1984, P1.40

(17) Georges de La Tour, Le Tricheur, Paris: Musée du Louvre. Thuillier, XXVIII-XXIX. La Diseuse de Bonne Aventure, New York: Metropoli$\tan$ Museum. Thuillier, XX-XXI.

(18) Le Caravage, Diseuse de Bonne Aventure, Paris: Musée du Louvre, Bonsanti, II.

(19) Léonard, $50, \mathrm{n}^{\circ} 90$.

(20) Georges de La Tour, Le Souffleur à la Lampe, Dijon: Musée des BeauxArts, Thuillier, Pl. XXXIII. 